\title{
Eye- and neck/shoulder-discomfort during visually demanding experimental near work
}

\author{
Camilla Lodin ${ }^{\mathrm{a},{ }^{*}}$, Mikael Forsman ${ }^{\mathrm{a}, \mathrm{b}}$ and Hans Richter ${ }^{\mathrm{a}}$ \\ ${ }^{a}$ Center for Musculoskeletal Research, Department of Occupational and Public Health Sciences, Faculty of \\ Health and Occupational Studies, University of Gävle, SE-801 76 Gävle, Sweden. \\ ${ }^{\mathrm{b}}$ Division of Occupational and Environmental Medicine, Department of Public Health Sciences, Karolinska \\ Institutet, SE-171 76 Solna, Sweden.
}

\begin{abstract}
Frequent use of digital information technology has an impact on eye- and neck/shoulder-discomfort. Studies with cross sectional and intervention design indicate an association between the two symptom categories. Still, whether visually demanding near work, per se, contributes to increased neck/shoulder discomfort remains a question of debate. The aim of this laboratory study was to assess if visually demanding experimental near work affects eye- and neck/shoulder-discomfort when the posture was adjusted for comfort and no movements were allowed. Thirty-three healthy subjects performed a visually demanding computer screen task (viewing task) under four different optical lens conditions: binocular $-3.5 \mathrm{D}$ and monocular $-3.5 \mathrm{D},+3.5 \mathrm{D}$ and $\pm 0 \mathrm{D}$. During the experiment subjects were seated in an office chair (with neck support) that was individually adjusted for comfort. At baseline and after each viewing task, subjects reported their perceived eye- and neck/shoulderdiscomfort on Borg's CR-10 scale. Results show a significant increase of eye discomfort between baseline and the first viewing task, and a significant increase in neck/shoulder discomfort from baseline throughout the first three viewing tasks. Further analysis is required to determent whether the neck/shoulder discomfort was induced by the demanding near work or the static posture, or a combination.
\end{abstract}

Keywords: Vision ergonomics, computer vision syndrome, eye strain, neck pain, Video Display Unit (VDU) users

\section{Introduction}

Professional users of modern information technology frequently report eye discomfort and/or neck/shoulder discomfort $[1,9,12]$. Many hours in front of the computer and oculomotor anomalies are often mentioned as factors influencing eye discomfort $[9,10]$. In contrast, forward bent neck posture, prolonged sitting, and static muscle activity are factors related to neck/shoulder discomfort [4].

There are studies from work places that indicate an association between eye discomfort and neck/shoulder discomfort [7, 11], and between physical symptoms and eye symptoms [6]. In studies with a cross sectional or intervention design, it is difficult to control all factors related to eye- or neck/shoulderdiscomfort, i.e. both the employee's posture throughout the workday and the visual load to which they are exposed. In order to evaluate the impact of visual load on eye- and neck/shoulder-discomfort without exposing them to unfavourable static postures in the neck, we designed the following laboratory study. The aim of this study was to assess if visually demanding experimental near work increases both eye discomfort and neck/shoulder discomfort when the posture was adjusted for comfort and no movements were allowed.

\footnotetext{
${ }^{*}$ Corresponding author: Camilla Lodin, University of Gävle, SE-801 76 Gävle, Sweden. E-mail: camilla.lodin.@hig.se
} 


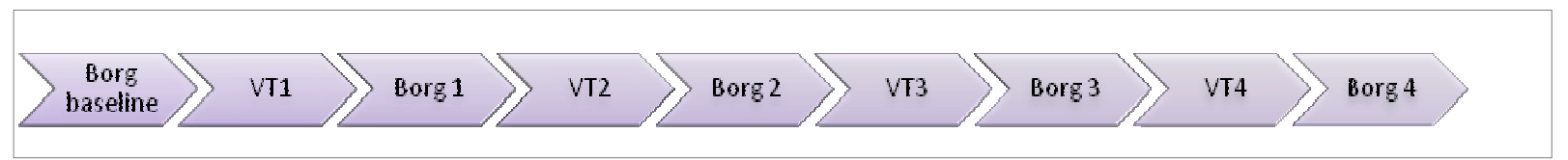

Fig. 1. Schematic representation of the experimental design. Each viewing task (VT1-VT4) consisted of three minutes rest with eyes closed and seven minutes focusing on a black-and white Gabor grating on a computer screen through one of four different optical trial lenses. Lens order was randomized using a Latin square. At baseline and after each viewing task, subject reported their perceived eye- and neck/shoulderdiscomfort on Borg's CR-10 scale.

\section{Methods}

This was a laboratory study. All subjects came to the lab on one occasion. In the lab a visually demanding viewing task was performed in front of a computer screen. The viewing task was performed four times, each utilising different optical-lenses mounted in trial frames.

\subsection{Subjects}

Thirty-three subjects without episodes of neck pain during the preceding three months were included in the study. Median age was 37 years (range 19-47), and six of the subjects were men. All subjects were volunteers, and the study was approved by the Uppsala University Medical Ethical Review board.

\subsection{Procedure}

In the lab, the subject was seated in a dark room on an office chair. Posture was individually adjusted for comfort and the head, neck, and back were supported. The subjects' upper arms hung alongside the trunk, and the hands were resting on the lap. To imitate visually demanding near work in this laboratory setting, a viewing task was developed. The subject did the viewing task four times (VT1-VT4) with different optical trial lenses mounted in trial frames.

Before the experiment started and after each viewing task, the subjects reported their perceived eye- and neck/shoulder-discomfort on Borg's CR-10 scale. The experimental design is illustrated in Figure 1.

\subsection{The viewing tasks}

The viewing task (VT) consisted of three minutes rest with eyes closed, followed by seven minutes of fixation. During each fixation the subject focused a black-and-white Gabor grating generated on a com- puter screen (Cambridge Research System) through one of four different trial-lenses. The grating had a fixation-cross in the middle - Figure 2. Distance to the screen was 65 centimeter (1.5 diopters (D)), gaze angel approximately $15^{\circ}$ downwards and the size of the image was $6.5 \times 6.5 \mathrm{~cm}$. The order of the triallenses was randomized among the subjects using a Latin square. In total, the subject's performed 28 minutes demanding near work (four fixations, $7 \mathrm{mi}$ nutes each).

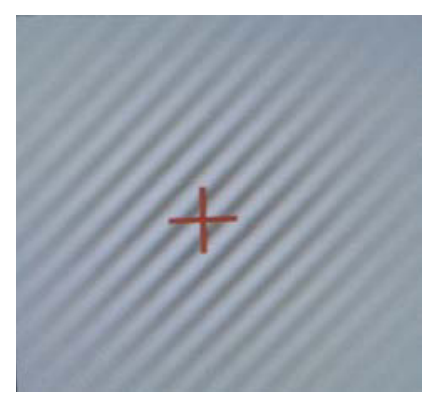

Fig. 2. The black-and-white Gabor grating subjects focused during the fixation task.

Before the fixation started, the spatial contrast $(\mathrm{C})$ $\left(\mathrm{C}=\left(\mathrm{L}_{\max }-\mathrm{L}_{\min }\right) /\left(\mathrm{L}_{\max }+\mathrm{L}_{\min }\right), \mathrm{L}=\right.$ luminance $)$ of the grating was zero and only the fixation-cross was visible. To start the task, the subject pushed a hand-held, low-force button and the contrast of the grating increased (speed 0.8 units/s.). When the subject perceived the grating, he/she pushed the button and the contrast froze for a short time. After the pause of random length (1.5-3.5 s.) the contrast of the grating decreased and when the grating was invisible to the subject, he/she pushed the button. This was repeated throughout the seven minutes fixation. Fixation instruction was: "Look at the fixation-cross and the black-and-white Gabor grating. Carefully focus on the fixation cross so that it is maximally sharp and clear at all times".

During the seven minutes fixation the subjects focused the grating through one of four different optical trial lenses: (i) binocular viewing through lenses with dioptric strength $-3.5 \mathrm{D}$; (ii) monocular viewing 
through $-3.5 \mathrm{D}$, (iii) monocular viewing through \pm 0 $\mathrm{D}$, and (iv) monocular viewing through +3.5 . In the monocular conditions, subject focused with their dominant eye and the non-dominant eye was occluded with a black lens. If the subject had refractive error, this was corrected for with spherical trial lenses. The stimuli (lens plus distance to screen) in condition (i) and (ii) was $5.0 \mathrm{D}$, in condition (iii) $1.5 \mathrm{D}$ and in condition (iv) $-2.0 \mathrm{D}$.

This study was carried out as part of a larger data collection. This part evaluates the effect of subsequent demanding viewing tasks on eye- and neck/shoulder-discomfort, and not the unique effect of different optical trial lenses.

\subsection{Amplitude of eye-lens accommodation}

To evaluate if subjects accommodated according to the instructions, an auto refractor (Power Refractor R03, Plusoptix, Nürnberg, Germany) recorded the crystalline eye-lens accommodation during the seven minutes fixation. Recorded accommodation was transformed into response diopters (cf. Richter et al. [8]) and compared to stimuli diopters.

To ensure recording from the auto refractor, the subjects' eyes had to be aligned to the measurement axis of the auto refractor. Therefore the subjects' were instructed to maintain the posture and to keep the head, neck, and back supported by the chair.

\subsection{Percived discomfort}

The subjects reported their perceived intensity of eye discomfort and neck/shoulder discomfort, using Borg's CR-10 scale, five times during the experiment - Figure 1 [2, 3]. Borg's CR-10 scale is a category ratio scale with written descriptions anchored to numbers - se figure 3. The question on eye discomfort was: Decide to what degree you feel pain or discomfort in your eyes. The question on neck/shoulder discomfort was: Decide to what degree you feel pain or discomfort in your neck and/or shoulders.

\begin{tabular}{|ll|}
\hline 0 & Nothing at all \\
0.5 & Extremely weak \\
1 & Very weak \\
2 & Weak \\
3 & Moderate \\
4 & \\
5 & Strong \\
6 & \\
7 & Very strong \\
8 & \\
9 & \\
10 & Extremely strong \\
& \\
\hline
\end{tabular}

Fig. 3. Borg's CR-10 scale with the written descriptors [2].

\subsection{Statistical analysis}

For statistical analysis PASW 18.0 for Windows was used (SPSS Inc., Chicago, IL, USA). Reported values on Borg's CR-10 scale were non-normally distributed, thus differences were analyzed using Wilcoxon signed rank test for repeated measurements of nonparametric data $(\alpha$-level 0.05$)$.

\section{Results}

\subsection{Amplitude of eye-lens accommodation}

Mean response diopters in condition (i) was $3.0 \mathrm{D}$ ( $\mathrm{sd}$ 2.0 ), in condition (ii) $3.3 \mathrm{D}$ (sd 1.8), in condition (iii) $1.4 \mathrm{D}$ ( $\mathrm{sd} 0.5$ ), and in condition (iv) $1.0 \mathrm{D}$ ( $\mathrm{sd} 0.8$ ).

\subsection{Eye discomfort}

The reported eye discomfort at baseline and after the subsequent viewing tasks (VT1-VT4) is presented in table 1 and figure 4 . There was a significant increase in eye discomfort after VT1 compared to baseline ( $\mathrm{z}$ $=-3.78, \mathrm{p}<0.001)$. After VT2 there was a decrease in eye discomfort, and thereafter the eye discomfort increased non-significantly. Average increase on group level between baseline and after VT4 was 1.70 .

Table 1. Ratings of eye discomfort on Borg's CR-10 scale at baseline and after each viewing task, VT1-VT4. N=33

\begin{tabular}{lllll}
\hline Eye discomfort & Mean & SD & Min & Max \\
\hline Baseline & 0.34 & 0.51 & 0 & 2 \\
VT1 & 1.48 & 1.69 & 0 & 6 \\
VT2 & 1.31 & 1.09 & 0 & 3 \\
VT3 & 1.69 & 1.50 & 0 & 6 \\
VT4 & 2.04 & 1.79 & 0 & 7 \\
\hline
\end{tabular}




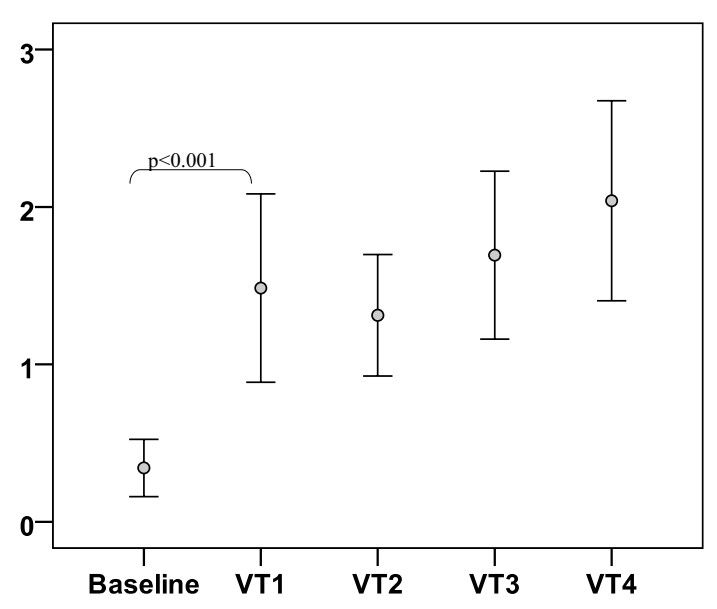

Fig. 4. Eye discomfort reported using Borg's CR-10 scale. Mean values and $95 \%$ confidence interval at baseline and after the subsequent viewing tasks, VT1-VT4. $\mathrm{N}=33$.

\subsection{Neck/shoulder discomfort}

The reported neck/shoulder discomfort at baseline and after the subsequent viewing tasks (VT1-VT4) is presented in table 2 and figure 5 . Mean values increased from baseline and all the way through the viewing tasks. The difference in neck/shoulder discomfort was significant after VT1 compared to baseline $(z=-2.68 p=0.007)$, after VT2 compared to VT1 $(z=-2.24, p=0.025)$, and after VT3 compared to VT2 $(\mathrm{z}=2.62, \mathrm{p}=0.009)$ - figure 5. Average increase on group level between baseline and VT4 was 1.62 .

Table 2. Ratings of neck/shoulder discomfort on Borg's CR-10 scale at baseline and after each viewing task 1-4. $\mathrm{N}=33$

\begin{tabular}{lllll}
\hline $\begin{array}{l}\text { Neck/shoulder } \\
\text { discomfort }\end{array}$ & Mean & SD & Min & Max \\
\hline Baseline & 0.48 & 0.69 & 0 & 2 \\
VT1 & 1.05 & 1.21 & 0 & 5 \\
VT2 & 1.32 & 1.06 & 0 & 4 \\
VT3 & 2.01 & 1.79 & 0 & 7 \\
VT4 & 2.10 & 1.32 & 0 & 5 \\
\hline
\end{tabular}

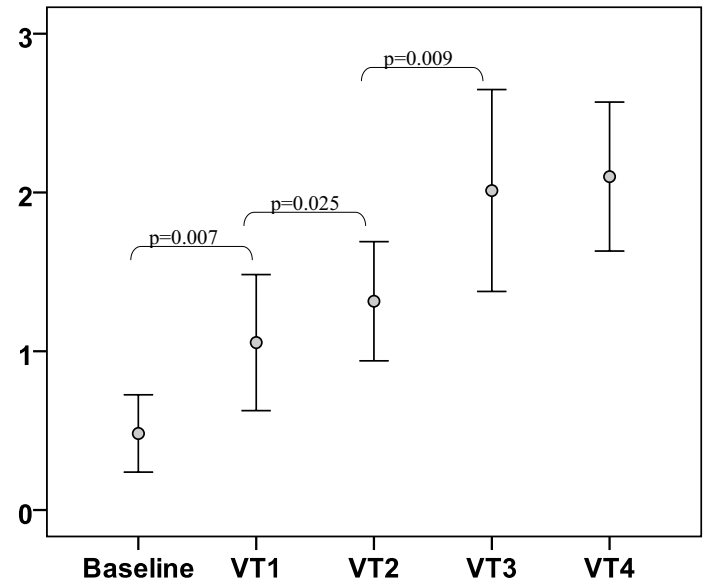

Fig. 5. Neck/shoulder discomfort reported using Borg's CR-10 scale. Mean values and $95 \%$ confidence interval at baseline and after the subsequent viewing tasks, VT1-VT4. $\mathrm{N}=33$.

\section{Discussion}

The aim of this study was to assess if simulated visually demanding near work increases both eye discomfort and neck/shoulder discomfort even though the posture was adjusted for comfort

The results revealed that both eye- and neck/shoulder-discomfort increase during visual demanding near work. The increase of eye discomfort was significant between baseline and the first viewing task (VT1). After viewing task two (VT2) there was a slight decrease in eye discomfort, and thereafter the increase was more moderate. Neck/shoulder discomfort increased significantly from baseline throughout the first three viewing tasks, but after the third viewing task the increase was not significant.

The subjects' had difficulties to meet the demands with the $-3.5 \mathrm{D}$ and $+3.5 \mathrm{D}$ lenses and it is likely that these lens-conditions were too demanding to some of the subjects. The expected accommodation ability of a 47 year old person is $4.4 \mathrm{D}$ (predicted value from Hofstetter's equation) and the accommodation required in the -3.5 lens conditions were $5 \mathrm{D}$. That means that at least the oldest subjects had problem to overcome the blur and to see the image clear with the minus lenses. Nevertheless, it is clear that the subjects did accommodate and that the task was visually demanding.

In comparison to a realistic occupational situation, the visual load in the minus conditions was high. 
Five diopters equals a viewing distance of $20 \mathrm{~cm}$ and for most people doing near work, e.g. computer work, the visual target (screen) is at least $40 \mathrm{~cm}$ away. On the other hand, the experimental load only lasts for in total 28 minutes, while working at a computer can continue many hours.

The increased eye discomfort is in line with previous findings. A survey among computer operators in India found that $46.3 \%$ of the subjects reported asthenopic symptoms including eyestrain and ocular discomfort [1]. Chu et al. [4] found that subjects rated higher visual symptoms after demanding near work at a computer screen, compared to reading a hard copy with identical text, size of letters, and contrast.

It turned out to be difficult to control the subjects' posture. The subjects were seated in a normal office chair adjusted for comfort in a slightly leaned back position, with head, neck, and back support. Due to the camera, subjects were instructed to maintain the same posture during the vision tasks, and they were also instructed to be relaxed. The test leader sat behind a screen and could only see the subjects' eyes through the camera. Therefore, minor adjustments of the neck posture that subjects did during the fixation tasks, e.g. protraction or flexion, were not detected.

Under experimental conditions, as in this study, selfreported eye- and neck/shoulder-discomfort increase during visual demanding near work, even when the seated posture has been adjusted for comfort. The reported eye discomfort was most likely induced by the visual load, however, under the presented study design, it can not be concluded whether if the neck/shoulder discomfort was induced by the visual load or the static posture. This experiment also included continuous sampling of ocular accommodation and trapezius electromyography. Analyses of the ocular accommodation and electromyography data together with the discomfort scores may clarify the root of the neck/shoulder discomfort. Should such analyses fail to clarify the relationship, further experimentation at a low, neutral, ocular load under the same posture and timing constraints should be undertaken.

\section{References}

[1] D. J. Bhanderi, S. Choudhary \& V. G. Doshi, A communitybased study of asthenopia in computer operators, Indian J Ophthalmol 56 (2008), 51-5.

[2] G. Borg, Psychophysical scaling with applications in physical work and the perception of exertion, Scand J Work Environ Health 16 Suppl 1 (1990), 55-8.

[3] G. A. Borg, Psychophysical bases of perceived exertion, Med Sci Sports Exerc 14 (1982), 377-81.

[4] B. Cagnie, L. Danneels, D. Van Tiggelen, V. De Loose \& D. Cambier, Individual and work related risk factors for neck pain among office workers: a cross sectional study, Eur Spine J 16 (2007), 679-86.

[5] C. Chu, M. Rosenfield, J. K. Portello, J. A. Benzoni \& J. D. Collier, A comparison of symptoms after viewing text on a computer screen and hardcopy, Ophthalmic Physiol Opt 31 (2011), 29-32.

[6] J. R. Hayes, J. E. Sheedy, J. A. Stelmack \& C. A. Heaney, Computer use, symptoms, and quality of life, Optom Vis Sci 84 (2007), 738-44.

[7] M. Helland, G. Horgen, T. M. Kvikstad, T. Garthus, J. R. Bruenech \& A. Aaras, Musculoskeletal, visual and psychosocial stress in VDU operators after moving to an ergonomically designed office landscape, Appl Ergon 39 (2008), 284-95.

[8] H. O. Richter, T. Banziger, S. Abdi \& M. Forsman, Stabilization of gaze: a relationship between ciliary muscle contraction and trapezius muscle activity, Vision Res 50 (2010), 2559-69.

[9] M. Rosenfield, Computer vision syndrome: a review of ocular causes and potential treatments, Ophthalmic Physiol Opt 31 (2011), 502-15.

[10]E. P. Rossignol Am Fau - Morse, V. M. Morse Ep Fau Summers, L. D. Summers Vm Fau - Pagnotto \& L. D. Pagnotto, Video display terminal use and reported health symptoms among Massachusetts clerical workers,

[11]C. Wiholm, H. Richter, S. E. Mathiassen \& A. Toomingas, Associations between eyestrain and neck-shoulder symptoms among callcenter operators, SJWEH suppl 3 (2007), 54-59.

[12] V. Woods, Musculoskeletal disorders and visual strain in intensive data processing workers, Occup Med , (2005), 9. 